BULL. AUSTRAL. MATH. SOC.

MOS $* 7620,3514,7635,7641,7665$

VOL. $2(1970), 429-431$.

\title{
Wave propagation in weakly nonlinear dispersive systems
}

\author{
Gordon V. Miles
}

The recent Discussion Meeting of the Royal Society, culminating several years of increased interest in the nonlinear dispersion of waves, posed questions about the possibility of many-valued and singular solutions, and about the validity and applicability of results. These, and other problems associated with the interrelationship between the theories of Phillips [7], Benjamin and Feir [1], Benney and Newell [2], Lighthill [4], [5] and Whitham $[8,9],[10,11]$, are discussed here using model equations, based on those of Bretherton [3] and Luke [6]. These models apparently describe the behaviour of physical systems without unnecessary algebraic encumbrances.

A general condition for the Benjamin and Feir instability is proposed for any weakly nonlinear wave system with a dispersion relation. The instability mechanism for a model system is followed until the imperceptible initial modulations become comparable with the wave amplitudes. The method could be applied to physical systems but the algebra would be much more unwieldly.

The same model facilitates examining closure interactions of finite wave packets. The group velocity separation destroys the periodic nature of discrete interactions, but locally energy transfer proceeds as if the wave trains were infinite. An unexpected sensitivity to the initial conditions is displayed.

Multiscaling methods are used to investigate propagation problems when continuous wavenumber spectra exclude discrete modal theories. The separate and combined effects of dispersion from wavenumber and amplitude variations, and their interdependence with the nonlinear effects are

Received 18 February 1970. Thesis submitted to the University of Western Australia, July 1969. Degree approved, January 1970. Supervisors: Professor H.C. Levey, Professor J.J. Mahony. 429 
discussed. It is shown how the inclusion of certain dispersive terms, in regions where energy is concentrated, prevents singularities of the approximate equations. The motion in concentration zones is governed by an equation of Benney and Newell; the importance of which, in cases other than the special balance of Benney and Newell [2], has not previously been recognised. This only yields a qualitative picture of the propagation because the Benney and Newell equation is not amenable to analytic solution. Consequently an alternative matching approach, which is consistent with the implicit assumptions of multiscaling, is proposed for weakly nonlinear cases. Numerical stability is assured by including the same dispersion terms needed to control the physical solution in concentration zones, and the Lighthill-Whitham propagation classification vanishes. Apparently this is true also for fully nonlinear systems.

\section{References}

[1] T. Brooke Benjamin and J.E. Feir, "The disintegration of wave trains on deep water", J. Fluid Mech. 27 (1967), 417-430.

[2] D.J. Benney and A.C. Newe I,, "The propagation of nonlinear wave envelopes", J. Math. and Phys. 46 (1967), 133-139.

[3] F.P. Bretherton, "Resonant interactions between waves. The case of discrete oscillations", J. Fluid Mech. 20 (1964), 457-479.

[4] M.J. Lighthill, "Contributions to the theory of waves in nonlinear dispersive systems", J. Inst. Math. App Z. 1 (1965), 269-306.

[5] M.J. Lighthill, "Some special cases treated by the Whitham theory", Proc. Roy. Soc. Ser. A 299 (1967), 28-53.

[6] J.C. Luke, "A perturbation method for nonlinear dispersive wave problems", Proc. Roy. Soc. Ser. A 292 (1966), 403-412.

[7] O.M. Phillips, "Theoretical and experimental studies of gravity wave interactions", Proc. Roy. Soc. Ser. A 299 (1967), 104-119.

[8] G.B. Whitham, "Non-linear dispersive waves", Proc. Roy. Soc. Ser. A 283 (1965), 238-261. 
[9] G.B. Whitham, "A general approach to linear and non-linear dispersive waves using a Lagrangian", J. Fluid Mech. 22 (1965), 273-283.

[10] G.B. Whitham, "Non-linear dispersion of water waves", J. Fluid Mech. 27 (1967), 399-412.

[11] G.B. Whitham, "Variational methods and applications to water waves", Proc. Roy. Soc. Ser. A 299 (1967), 6-25. 